\title{
Photon Momentum Sharing between an Electron and an Ion in Photoionization: From One-Photon (Photoelectric Effect) to Multiphoton Absorption
}

\author{
Szczepan Chelkowski, ${ }^{1, *}$ André D. Bandrauk, ${ }^{1}$ and Paul B. Corkum ${ }^{2}$ \\ ${ }^{1}$ Laboratoire de Chimie Théorique, Faculté des Sciences, Université de Sherbrooke, Sherbrooke, Québec, Canada J1K $2 R 1$ \\ ${ }^{2}$ Joint Attosecond Science Laboratory of the National Research Council and the University of Ottawa, \\ 100 Sussex Drive, Ottawa, Ontario, Canada K1A 0R6
}

(Received 28 July 2014; published 31 December 2014)

\begin{abstract}
We investigate photon-momentum sharing between an electron and an ion following different photoionization regimes. We find very different partitioning of the photon momentum in one-photon ionization (the photoelectric effect) as compared to multiphoton processes. In the photoelectric effect, the electron acquires a momentum that is much greater than the single photon momentum $\hbar \omega / c$ [up to $(8 / 5)$ $\hbar \omega / c]$ whereas in the strong-field ionization regime, the photoelectron only acquires the momentum corresponding to the photons absorbed above the field-free ionization threshold plus a momentum corresponding to a fraction $(3 / 10)$ of the ionization potential $I_{p}$. In both cases, due to the smallness of the electron-ion mass ratio, the ion takes nearly the entire momentum of all absorbed $N$ photons (via the electron-ion center of mass). Additionally, the ion takes, as a recoil, the photoelectron momentum resulting from mutual electron-ion interaction in the electromagnetic field. Consequently, the momentum partitioning of the photofragments is very different in both regimes. This suggests that there is a rich, unexplored physics to be studied between these two limits which can be generated with current ultrafast laser technology.
\end{abstract}

DOI: 10.1103/PhysRevLett.113.263005

PACS numbers: $32.80 . \mathrm{Rm}, 32.80 . \mathrm{Gc}, 32.80 . \mathrm{Wr}$

In a recent experiment [1] the average photoelectron momentum component along the laser propagation (which we call parallel, $\mathrm{Oz}$ direction) was measured in the process involving absorption of many, circularly polarized (30-50) photons. The experiment showed that this component, $p_{z}^{e}$, of the electron momentum grows linearly as a function of the laser intensity. More specifically, it was shown that $p_{z}^{e}$ is nearly equal to the average photoelectron energy divided by the speed of light. Assuming that the number of photons absorbed is the number needed to overcome the ionization potential $I_{p}$ plus the number required to supply to the above threshold ionization, ATI, the above finding means that the momentum associated with $I_{p}$ is absent from the electron momentum; it therefore must be given to the ion. The experimental results can be interpreted as the breakdown of the dipole approximation at surprisingly low light intensities $\left(I=10^{14} \mathrm{~W} / \mathrm{cm}^{2}\right)$ where the electron energies are far from the relativistic regime and the laser wavelength $\lambda$ is much longer than the characteristic atomic size $2 a_{0}=0.106 \mathrm{~nm}$. The dipole approximation is commonly used up to much higher intensities, much greater than $I=10^{14} \mathrm{~W} / \mathrm{cm}^{2}[2,3]$. Within this approximation the electron does not gain any net momentum along the laser propagation direction $(\mathrm{Oz})$; i.e., the dipole approximation predicts that the distribution $f\left(p_{z}^{e}\right)$ of the electron momentum along the propagation direction is symmetric with respect to $p_{z}^{e}=0$ and consequently the average momentum $\left\langle p_{z}^{e}\right\rangle=0$. So far, to our knowledge, in nearly all current theoretical, beyond-dipole-approximation approaches [4-7], the issue of sharing of the momentum between an electron and an ion was not addressed in detail since in most previous theoretical work the center of mass motion was not considered at all and the theory was formulated in terms of only the relative electron-ion coordinate and relative electron-ion momentum. Thus, the total transfer of the photon's momentum to the atom has not been taken into account. The only exception is a [8] Monte Carlo classical model for the electron. However, such a model relies on the unknown distribution of initial conditions for the classical trajectories launched after tunneling of the electron from an initial quantum bound state.

We demonstrate how the photon momentum is shared between the electron and the ion in the case of single photon absorption, the perturbation regime, and in the case of the multiphoton absorption, the nonperturbative regime. For the former case we use perturbation theory whereas for the latter we extend the strong field approximation (SFA) $[2,3]$ method and use a time dependent Gaussian pulse envelope. In both cases, we include the electron and ion degrees of freedom. These calculations suggest the following physical insights:

(i) For the one-photon absorption case:

$$
\left\langle p_{z}^{e}\right\rangle=(8 / 5) E_{\mathrm{el}} / c, \quad\left\langle p_{z}^{i}\right\rangle=-\frac{3}{5} \frac{E_{\mathrm{el}}}{c}+\frac{I_{p}}{c},
$$

where $E_{\mathrm{el}}=\hbar \omega-I_{p}$ is the kinetic energy of the photoelectron. The presence of the $(8 / 5)$ factor is surprising: it leads to the conclusion [4] that when $\hbar \omega>(8 / 3) I_{p}$ we 
have $\left\langle p_{z}^{e}\right\rangle>\hbar \omega / c$ which means that the photoelectron takes more momentum than the photon possesses. Equation (1) is one important result of our calculation.

(ii) For multiphoton, strong-field, ionization with low photon frequencies, $\hbar \omega \ll I_{p}$, we find

$$
\left\langle p_{z}^{e}\right\rangle=\left\langle E_{\mathrm{el}}\right\rangle / c+0.3 I_{p} / c, \quad\left\langle p_{z}^{i}\right\rangle=0.7 I_{p} / c,
$$

where $\left\langle E_{\mathrm{el}}\right\rangle$ is the photoelectron energy averaged over the whole ATI spectrum. In both Eqs. (1) and (2) the electron momentum results from the dynamics occurring in the relative coordinates and relative momenta. Therefore, the same momentum is taken by an ion in the opposite direction via recoil. The ion also acquires, via c.m. motion, nearly the entire momentum of $n$-absorbed photons which results in the net ion momentum $0.7 I_{p} / c$. This ion momentum is very different from the net ion momentum, Eq. (1), in the case of the photoelectric effect which for large photon energies becomes $-(3 / 5) \hbar \omega / c$.

(iii) We note that in the multiphoton regime a surprising $0.3 I_{p} / c$ shift in the electron parallel momentum appears. While this term did not appear in Ref. [1], the relation $\left\langle p_{z}^{e}\right\rangle=\left\langle E_{\mathrm{el}}\right\rangle / c+0.3 I_{p} / c$ is not inconsistent with the experimental results [1] because of the experimental errors which are close to $0.3 I_{p} / c$. A similar shift appears in a relativistic tunneling model presented in Ref. [7] but is absent in other approaches $[5,6]$.

(iv) With the net momenta of the electron and of the ion, very different in these two extremes, one is forced to conclude that there must be a rich and complex physics that links the two. This physics has never been explored.

The two body electron-nucleus quantum Hamiltonian in the Coulomb gauge $(\nabla \cdot \vec{A}=0$ where $\vec{A}$ is the vector potential of a classical electromagnetic, EM, field) is written as

$$
\hat{H}\left(\hat{\vec{p}}_{e}, \hat{\vec{p}}_{i}, \vec{r}_{e}, \vec{r}_{i}, t\right)=\hat{H}_{0}+\hat{V}_{\mathrm{int}}^{e}\left(t, \vec{r}_{e}\right)+\hat{V}_{\mathrm{int}}^{i}\left(t, \vec{r}_{i}\right)
$$

with the free atom Hamiltonian $\hat{H}_{0}$ :

$$
\hat{H}_{0}=\frac{1}{2 m_{e}} \hat{\vec{p}}_{e}^{2}+\frac{1}{2 m_{i}} \hat{\vec{p}}_{i}^{2}-\frac{e^{2} Z}{\left|\vec{r}_{e}-\vec{r}_{i}\right|},
$$

and the time dependent field interactions with an electron and a nucleus having charges $-e(e>0$ is the elementary charge) and $Z e$, respectively,

$$
\hat{V}_{\mathrm{int}}^{e}\left(t, \vec{r}_{e}\right)=\frac{e}{m_{e} c} \vec{A}\left(\vec{r}_{e}, t\right) \cdot \hat{\vec{p}}_{e}+\frac{e^{2}}{2 m_{e} c^{2}} \vec{A}^{2}\left(\vec{r}_{e}, t\right) .
$$

$\hat{\vec{p}}_{e}$ and $\hat{\vec{p}}_{i}$ are momentum operators of the electron and the ion, respectively. We neglect the interaction $\hat{V}_{\text {int }}^{i}$ of the EM field with the ion. We have checked that this term does not modify the momenta distribution $f\left(p_{z}\right)$ and averages $\left\langle p_{z}^{e}\right\rangle$ plotted in all figures because of the smallness of the electron-ion mass ratio, $m_{e} / m_{i}$. We separate the center of mass motion in $\hat{H}_{0}$ via a standard transformation

$\vec{R}_{\mathrm{c} . \mathrm{m} .}=\left[m_{e} \vec{r}_{e}+m_{i} \vec{r}_{i}\right] / M_{t}, \quad \vec{r}=\vec{r}_{e}-\vec{r}_{i}, \quad M_{t}=m_{e}+m_{i}$,

$$
\hat{\vec{P}}_{\text {c.m. }}=\hat{\vec{p}}_{e}+\hat{\vec{p}}_{i}, \quad \hat{\vec{p}}=\left[m_{i} \hat{\vec{p}}_{e}-m_{e} \hat{\vec{p}}_{i}\right] / M_{t} .
$$

Thus $\hat{H}_{0}$ describes the c.m. motion as a free particle and the motion (in the relative coordinate) of a "particle" having electron-nucleus reduced mass $m_{e}^{\prime}=m_{e} m_{i} / M_{t}$. By contrast, such a separation is impossible when the interaction $\hat{V}_{\text {int }}^{e}\left(t, \vec{r}_{e}\right)$ term is added since the term $\vec{A}\left(t-z^{e} / c\right) \cdot \vec{p}^{e}$ couples (via relation $z^{e} \simeq z+Z_{\text {c.m. }}$.) the c.m. motion with the relative motion.

We calculate the photoelectron momentum $f\left(p_{z}\right)$ distributions in the case of the simple photoelectric effect for UV (or XUV) frequencies (i.e., when $\hbar \omega>I_{p}$ and the laser intensity is weak). We use the standard time dependentperturbation theory which gives the following first order transition amplitude for a transition from an initial state $\psi_{\text {in }}$ to the final state $\psi_{f}$ [9]:

$A_{f i} \simeq \frac{-i}{\hbar} \int_{-\infty}^{\infty} d t\left\langle\psi_{f}\left|e^{i \hat{H}_{0} t / \hbar} \hat{V}_{\mathrm{int}}^{e}\left(t-z_{e} / c\right) e^{-i \hat{H}_{0} t / \hbar}\right| \psi_{\mathrm{in}}\right\rangle$.

First, we use linear polarization along the $x$ axis and a Gaussian form of the laser pulse envelope in $\vec{A}\left(t^{\prime}\right)$ :

$$
\vec{A}\left(t^{\prime}\right)=-\vec{e}_{x} \frac{c}{\omega} E_{0} \exp \left[-\frac{t^{\prime 2}}{2 \tau_{G}^{2}}\right] \sin \left(\omega t^{\prime}\right),
$$

where $t^{\prime}=t-z_{e} / c$ is the retardation time. $E_{0}, \vec{e}_{x}, \omega$, and $\tau_{G}$ are the UV pulse amplitude of the electric field, polarization vector, its central frequency, and duration. Our initial state is

$$
\psi_{\text {in }}=(2 \pi \hbar)^{-3 / 2} \psi_{B}(\vec{r}) \int d^{3} P \Phi_{\text {c.m. }}(\vec{P}) \exp \left(\frac{i}{\hbar} \vec{R}_{\text {c.m. }} \cdot \vec{P}\right)
$$

where $\Phi_{\text {c.m. }}(\vec{P})$ describes the momentum spread of the initial center-of-mass (c.m.) wave packet. It is assumed that the average of the initial c.m. momentum $\left\langle\vec{P}_{\mathrm{c} . \mathrm{m} .}\right\rangle=0$ and that the norm of $\Phi_{\text {c.m. }}\left(\vec{P}_{\text {c.m. }}\right)$ is equal to 1 . The final state is

$$
\psi_{f}=(2 \pi \hbar)^{-3 / 2} \exp \left(\frac{i}{\hbar} \vec{P}_{\text {c.m. }}^{f} \cdot \vec{R}_{\text {c.m. }}\right) \psi_{\vec{p}}^{c}(\vec{r}),
$$

where $\psi_{\vec{p}}^{c}(\vec{r})$ is the Coulomb wave. We next replace the exact Coulomb wave $\psi_{\vec{p}}^{c}(\vec{r})$ by a plane wave $(2 \pi \hbar)^{-3 / 2} \exp (i \vec{p} \cdot \vec{r} / \hbar)$ which allows us to obtain simple analytic formulas. We show in Ref. [10] that the distribution of momenta $f\left(p_{z}\right)$ and the average momentum $\left\langle p_{z}\right\rangle$ are not affected by the Coulomb corrections in the case of the 
initial $1 s$ bound state and we also provide there more details for the derivation of the momenta distribution $f\left(p_{z}^{e}\right)$. Since the interaction $V_{\mathrm{int}}^{e}$ depends on the retardation $t^{\prime}=t-z_{e} / c$, we change in Eq. (8) the integration variable $t$ into $t^{\prime}=t-z_{e} / c$ and we use the relation $z_{e}=Z_{\text {c.m. }}+\alpha^{\prime} z$ where $\alpha^{\prime}=m_{i} / m_{t}$. We thus get

$A_{f i}=\frac{2 e E_{0} p_{x} g(x)}{\sqrt{\pi} m_{e} \hbar \omega} \tilde{\tilde{r}_{B}}\left(\vec{p}-\alpha^{\prime} K \vec{e}_{z}\right) \Phi_{\text {c.m. } .}\left(\vec{P}_{\text {c.m. }}^{f}-K \vec{e}_{z}\right)$,

where $\tilde{\psi}_{B}(\vec{p})=(8 / \pi)^{1 / 2} p_{a}^{5 / 2}\left[p_{a}^{2}+\vec{p}^{2}\right]^{-2}$ is the Fourier transform of the initial bound, $1 s$, state which in the case of a H-like ion, $p_{a}=Z \hbar / a_{0}, a_{0}$ is the Bohr radius and $g(x)=\sqrt{2 \pi} \tau_{G} \exp \left[-\tau_{G}^{2} x^{2} / 2 \hbar^{2}\right]$ is the Fourier transform of the Gaussian laser pulse envelope and $x=E_{f}+I_{p}-\hbar \omega$. $g(x)$ for long pulses becomes $2 \pi \hbar \delta\left(E_{f}+I_{p}-\hbar \omega\right)$. For long monochromatic pulses $K=\left(E_{f}+I_{p}\right) / c$ becomes equal to the photon momentum $\hbar \omega / c$. Note that the initial c.m. momentum distribution $\Phi_{\text {c.m. }}\left(\vec{P}_{\text {c.m. }}\right)$ is shifted by the photon momentum $K$ which means that the c.m. acquires exactly the photon momentum $\hbar \omega / c$. Additionally, the initial (relative) bound state momentum distribution in $\tilde{\psi}_{B}(\vec{p})$ is also shifted by the momentum of nearly the same momentum $=\alpha^{\prime} K \vec{e}_{z}$. Next we calculate the average relative momenta along the $z$ axis, see details in Ref. [10]. After inverting Eq. (7) we obtain

$$
\begin{gathered}
\left\langle p_{z}^{e}\right\rangle=\left\langle p_{z}\right\rangle+\frac{m_{e}}{M_{t}}\left\langle P_{z}^{\text {c.m. }}\right\rangle \simeq \frac{8}{5} \frac{E_{\mathrm{el}}}{c}, \\
\left\langle p_{z}^{i}\right\rangle=-\left\langle p_{z}\right\rangle+\frac{m_{i}}{M_{t}}\left\langle P_{z}^{\text {c.m. }}\right\rangle \simeq-\frac{3}{5} \frac{E_{\mathrm{el}}}{c}+\frac{I_{p}}{c} .
\end{gathered}
$$

In Fig. 1 we show the momenta $\left\langle p_{z}^{e}\right\rangle$ and $\left\langle p_{z}^{i}\right\rangle$, as a function of the electron energy, calculated using more exact expressions derived in Ref. [10] in which the continuum Coulomb

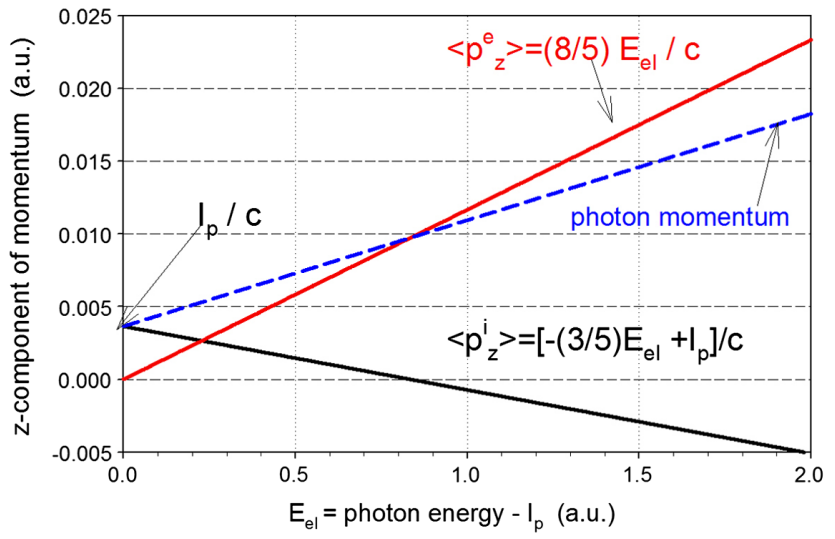

FIG. 1 (color online). Electron and ion average momenta along the propagation direction $(\mathrm{Oz})$ as a function of the electron energy $E_{\mathrm{el}}=\hbar \omega-I_{p}$ for a $\mathrm{H}$ atom in the UV-laser weak field (photoelectric effect). wave function is included and the integrals over momenta are performed numerically. The most important conclusions are as follows: When the photoelectron energy approaches zero, i.e., when $E_{\mathrm{el}} \ll I_{p}$ the ion takes the momentum equal to $\left(m_{i} / M_{t}\right) I_{p} / c$ whereas the electron takes the much smaller part equal to $\left(m_{e} / M_{t}\right) I_{p} / c$. By contrast, for large photon frequencies, $\hbar \omega>(8 / 3) I_{p}$, the electron acquires more momentum than the photon possesses [4]. We note that the photon momentum $K=\hbar \omega / c$ is shared unequally for all photoionization processes; i.e., nearly the entire photon momentum $K$ is directly transferred (via c.m. momentum) to the nucleus, see Eq. (14). However, both the electron and ion acquire momentum $\left\langle p_{z}\right\rangle$ and $-\left\langle p_{z}\right\rangle$, respectively. These additional opposing momenta do not originate directly from the photon momentum but arise from the (beyond-dipole approximation) internal dynamics electron-ion dynamics via Coulomb attraction induced by the magnetic force from the laser magnetic field. Thus any (average) electronmomentum increase in $\mathrm{Oz}$ direction is compensated by the (average) nucleus recoil in the opposite direction. It is easy to show that the same momenta distributions $f\left(p_{z}\right)$ are valid for the circular polarization as well: it suffices to replace $p_{x}$ by $p_{x} \pm i p_{y}$ and redo the integration over the momenta $p_{x}$ and $p_{y}$ in Eq. (12).

The relation, Eq. (13), containing the surprising factor $(8 / 5)$ leading to the large photon momentum transfer was first found by Michaud and next by Seaton [4]. They obtained it using Eq. (72.10) given in Ref. [16]. We show in Ref. [10] (at the end of Appendix 1) that our amplitude, Eq. (12), leads to the same angular distributions. Michaud and Seaton further emphasize the unusual discovery that (for large photon energies) the electrons go away with more momentum than the photons brought in. To our knowledge, all earlier and later work related to the retardation in the photoelectric effect missed this effect since it focused on the shift of a maximum of the electron angular distributions instead of on the direct average photon momentum transfer $\left\langle p_{z}^{e}\right\rangle$.

We now include the center of mass motion and photon momentum into the standard SFA approach as reviewed in Ref. [3] in which the transition amplitude (within the dipole approximation) is expressed via a Volkov wave function $\psi_{V}$ which replaces the simple plane waves in the transition amplitude, Eq. (8). $\psi_{V}$, as usually used in SFA [3], is the exact wave function for an electron interacting with an electromagnetic wave only in the case when one neglects the electron coordinate inside the vector potential $\vec{A}$, i.e., within the dipole approximation. In our approach, using the (relativistic) Klein-Gordon Volkov wave function [17] we obtain the following (beyond-dipole approximation, nonrelativistic) Volkov wave function [10]:

$$
\psi_{V}^{\mathrm{bd}}\left(\vec{r}_{e}, t\right)=\frac{1}{(2 \pi \hbar)^{3 / 2}} \exp \left[\frac{i}{\hbar}\left[\vec{p}_{e} \cdot \vec{r}_{e}-E_{\mathrm{el}} t-S_{\mathrm{bd}}\left(\vec{p}_{e}, t^{\prime}\right)\right]\right],
$$




$$
\begin{aligned}
S_{\mathrm{bd}}\left(\vec{p}_{e}, t^{\prime}\right) & =G\left(p_{z}^{e}\right) \int_{-\infty}^{t^{\prime}} d \tau^{\prime} V_{\mathrm{int}}^{e}\left(\tau^{\prime}\right), \\
G\left(p_{z}^{e}\right) & =1+p_{z}^{e} /\left(m_{e} c\right),
\end{aligned}
$$

where $t^{\prime}=t-z_{e} / c$. The factor $G\left(p_{z}^{e}\right)$ originates from the relativistic scalar product $\mathbf{k} \cdot \mathbf{p}_{e}$ present in the relativistic Volkov wave function [18,19], see details in Ref. [10]. The nondipole SFA transition amplitude becomes

$$
\begin{gathered}
A_{f i}=\frac{-i}{\hbar} \int_{-\infty}^{\infty} d t\left\langle\psi_{f}\left|V_{\mathrm{int}}\left(t-z_{e} / c\right) e^{i I_{p} t / \hbar}\right| \psi_{\mathrm{in}}\right\rangle, \\
\psi_{f}=(2 \pi \hbar)^{-3} \exp \left[\frac{i}{\hbar}\left(\vec{p} \cdot \vec{r}+\vec{P}_{\mathrm{c} . \mathrm{m} .} \cdot \vec{R}_{\mathrm{c} . \mathrm{m} .}-E_{f} t-S_{\mathrm{bd}}\right)\right],
\end{gathered}
$$

where $E_{f}=p^{2} / 2 m_{e}^{\prime}+\left(P_{\text {c.m. }}^{f}\right)^{2} / 2 M_{t}$ is the final energy of an electron and an ion. We insert Eq. (18) into the amplitude given in Eq. (17) and change the integration $t$ variable into $t^{\prime}=t-z_{e} / c$ (as we did for the one-photon processes) which leads to the appearance of the electron spatial variable $z_{e}=Z_{\text {c.m. }}+\alpha^{\prime} z$ within the matrix element. Thus we obtain a new beyond-dipole approximation SFA amplitude:

$$
\begin{aligned}
& A_{f i}=\frac{-i}{\hbar} \tilde{\psi_{B}}\left(\vec{p}-K \vec{e}_{z}\right) \Phi_{\text {c.m. }}\left(\vec{P}_{\text {c.m. }}-K \vec{e}_{z}\right) \tilde{a}(\vec{p}, K), \\
& \tilde{a}(\vec{p}, K)=\int_{-\infty}^{\infty} d t^{\prime} V_{\text {int }}\left(t^{\prime}\right) \exp \left[\frac{i}{\hbar}\left[\left(E_{f}+I_{p}\right) t^{\prime}+S_{\text {bd }}\right]\right] .
\end{aligned}
$$

In standard SFA approaches, applied for monochromatic pulses, the integral present in Eq. (20) is performed analytically using the Fourier-Bessel expansion of the $\exp [i x \sin \omega t]$ term [3]. However, since we expect from classical calculations [1] the importance of using pulses with time-dependent envelopes, we perform numerically the time integral present in Eq. (20) using a Gaussian pulse envelope (with a pulse duration of 15 fs duration). Next we calculate the average electron and ion momenta along the $\mathrm{Oz}$ axis using Eqs. (1), (7)-(10) given in [10].

In Figs. 2 and 3 and in Fig. 1 in Ref. [10] we plot the average electron momenta $\left\langle p_{z}^{e}\right\rangle$ and ion momenta $\left\langle p_{z}^{i}\right\rangle$ using the extended beyond-dipole SFA amplitude (19). Circularly polarized pulses are used except in Fig. 1 in Ref. [10] in which linearly polarized pulses are also used (dashed lines). In Fig. 2 the electron momentum $\left\langle p_{z}^{e}\right\rangle$ and the average electron energy $\left\langle E_{\mathrm{el}}\right\rangle / c$ are plotted as a function of laser intensity for $\mathrm{Ne}$ atoms at $\lambda=800 \mathrm{~nm}$. Both depend linearly on the laser peak intensity and have the same slope. This suggests that we replot $\left\langle p_{z}^{e}\right\rangle$ in Fig. 3 as a function of the average electron energy $\left\langle E_{\mathrm{el}}\right\rangle$. We also show in Fig. $3\left\langle p_{z}^{e}\right\rangle$ obtained for a $\mathrm{Ne}$ with that for an

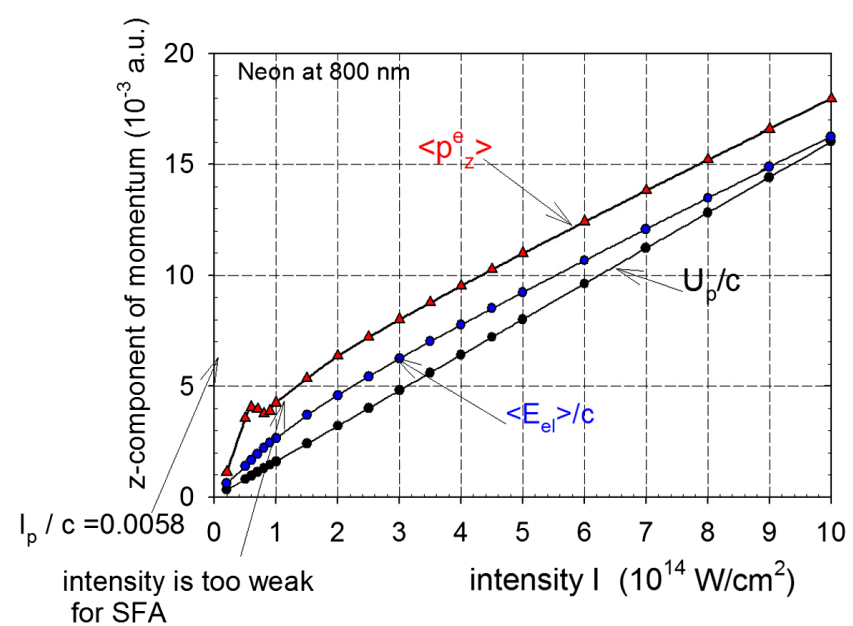

FIG. 2 (color online). (Triangles) Electron average momenta along the propagation direction $(\mathrm{Oz})$ obtained using the nondipole SFA model as a function of the laser intensity (circular polarization) for $\mathrm{Ne}$, are at $\lambda=800 \mathrm{~nm}$, compared with the average electron energy $\left\langle E_{\mathrm{el}}\right\rangle$ and with the ponderomotive energy $U_{p}=\left(e E_{0}\right)^{2} /\left(4 m_{e} \omega^{2}\right)$.

Ar atom at larger wavelength, $\lambda=1400 \mathrm{~nm}$ and in Fig. 1 in Ref. [10] for a $\mathrm{H}$ atom at $\lambda=800 \mathrm{~nm}$. Next we find that a following simple fit

$$
\left\langle p_{z}^{e}\right\rangle=\left\langle E_{\mathrm{el}}\right\rangle / c+0.3 I_{p} / c, \quad\left\langle p_{z}^{i}\right\rangle=0.7 I_{p} / c
$$

describes well the relations between $\left\langle p_{z}^{e}\right\rangle$ and average electron kinetic energy $\left\langle E_{\mathrm{el}}\right\rangle=\left\langle p_{e}^{2} / 2 m_{e}\right\rangle$ for all three cases shown in Fig. 3 and in Fig. 1 in Ref. [10]. We

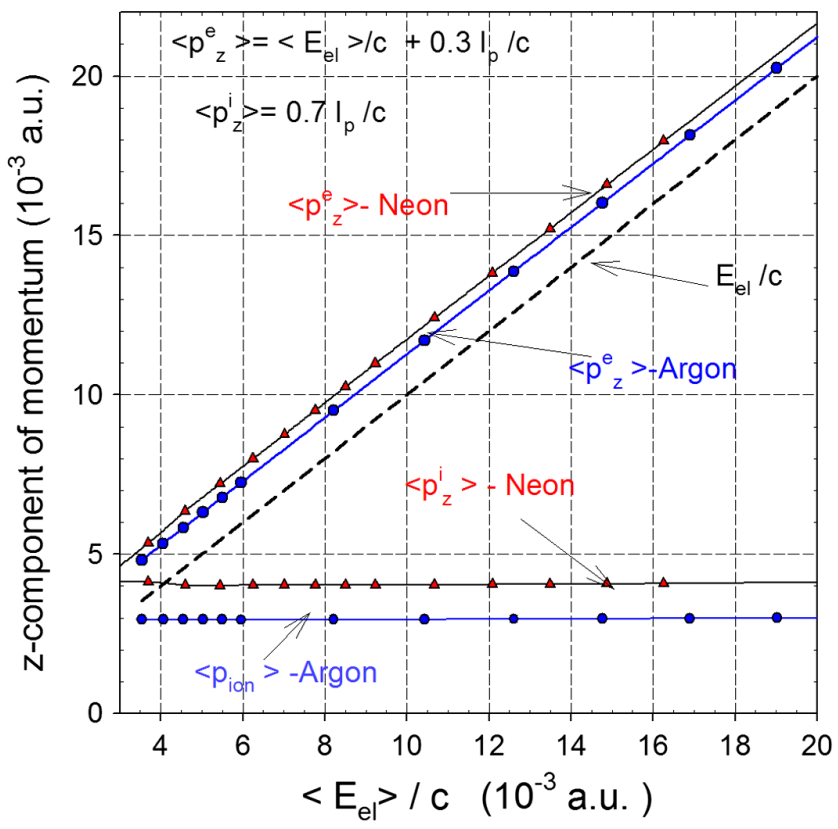

FIG. 3 (color online). Same as in Fig. 2 but with $\left\langle p_{z}^{e}\right\rangle$ for $\mathrm{Ar}$ at larger wavelength $\lambda=1400 \mathrm{~nm}$. Horizontal lines show the corresponding ion average momenta $\left\langle p_{z}^{i}\right\rangle$. 
emphasize that the rule given in Eq. (21) works very well for three different atoms, two wavelengths and for both, circular and linear polarizations, the latter is illustrated in Fig. 1 in Ref. [10] for a $\mathrm{H}$ atom. Figure 2 also shows the average ion momenta $\left\langle p_{z}^{i}\right\rangle$ as a function of $\left\langle E_{\mathrm{el}}\right\rangle / c$ calculated using the first term in Eq. (14) and the relation $\left\langle P_{\text {c.m. }}\right\rangle=\left(E_{\mathrm{el}}+I_{p}\right) / c$. Clearly, the ion momenta follow horizontal lines satisfying the simple universal relation $\left\langle p_{z}^{i}\right\rangle=0.7 I_{p} / c$ showing very different dependence on electron energy from the case of simple photoelectric effect, see Fig. 1, which shows that for large electron energy the ion momenta are negative and decrease linearly as a function of the electron energy. Figure 3 shows that the rule given in Eq. (21) even works for low electron energies $\left\langle E_{\mathrm{el}}\right\rangle$; however, this result can be unreliable since the SFA is only valid for $\left\langle E_{\text {el }}\right\rangle$ larger than $I_{p}$. We observe in Fig. 1 in Ref. [10] some deviations from the simple linear rule Eq. (21) at low electron energies for linear polarization. Note that the slope of one in the intense field, multiphoton regime is smaller than in the one-photon regime where slope equals $(8 / 5)$. Moreover, the shift by $0.3 I_{p} / c$ is absent in the 1-photon process, Eq. (1). We believe that this upward shift is related to the Lorentz-force experienced by the bound electron before (and during) the tunneling leading to the lowering of the tunneling rate in the $\mathrm{Oz}$ direction as discussed in Ref. [7].

Figures 2 and 3 show predictions of our extended (beyond the dipole approximation) SFA model for the laser pulse having a Gaussian envelope (9). In the standard SFA one uses a monochromatic pulse allowing us to use the expansion in which (for circular polarization) the Bessel functions $[3,10] J_{n}\left(x_{n}\right)$ appear. Using the relativistic Volkov wave function Eq. (15) leads to the following rescaling of the Bessel functions argument: $x_{n} \rightarrow G\left(p_{z}^{e}\right) x_{n}[18,19]$. This rescaling effectively lowers the tunneling rate of electrons which emerge in the beam propagation direction [7].

In conclusion, we have demonstrated the existence of universal relations describing the photon momentum sharing between an electron and ion in two specific, one-photon and multiphoton, regimes of atomic photoionization processes. These results suggest a set of interesting experiments for the future involving infrared or UV laser pulses, in which electron and ion momenta would be measured. In general, in intense fields a large transfer of photon momentum occurs to nuclei from the center of mass and cannot be neglected. Extending these new physical effects will also allow the photon induced nonadiabatic effects in molecules [20] and in molecular imaging by photoelectron ionization to be examined [21].

We thank NSERC for supporting Attosecond Science and also Compute Canada-Calcul Québec for access to supercomputers.

*S.Chelkowski@USherbrooke.ca

[1] C. T. L. Smeenk, L. Arissian, B. Zhou, A. Mysyrowicz, D. M. Villeneuve, A. Staudte, and P. B. Corkum, Phys. Rev. Lett. 106, 193002 (2011).

[2] D. B. Milosevic, G. G. Paulus, D. Bauer, and W. Becker, J. Phys. B 39, R203 (2006).

[3] J. Bauer, Phys. Rev. A 73, 023421 (2006).

[4] G. Michaud, Astrophys. J. 160, 641 (1970); M. J. Seaton, J. Phys. B 28, 3185 (1995).

[5] A. S. Titi and G. W. F. Drake, Phys. Rev. A 85, 041404(R) (2012).

[6] H. R. Reiss, Phys. Rev. A 87, 033421 (2013).

[7] M. Klaiber, E. Yakaboylu, H. Bauke, K. Z. Hatsagortsyan, and C. H. Keitel, Phys. Rev. Lett. 110, 153004 (2013).

[8] J. Liu, Q.Z. Xia, J. F. Tao, and L. B. Fu, Phys. Rev. A 87, 041403(R) (2013).

[9] A. Messiah, Quantum Mechanics (J. Wiley, New York, 1961), Vol. II.

[10] See Supplemental Material at http://link.aps.org/ supplemental/10.1103/PhysRevLett.113.263005, which includes Refs. [11-15], for details of the calculations of the average electron and ion momenta along the $\mathrm{Oz}$ axis and for the Coulomb field effect.

[11] L. Landau and E. Lifschitz, Quantum Mechanics (Pergamon Press, Section 113, 1958).

[12] G. L. Yudin, S. Patchkovskii, and A. D. Bandrauk, J. Phys. B 39, 1537 (2006), Eq. (29).

[13] A. Nordsieck, Phys. Rev. A 93, 785 (1954).

[14] N. J. Kylstra, R. M. Potvliege, and C. J. Joachain, J. Phys. B 34, L55 (2001).

[15] M. Verschl and C. H. Keitel, Laser Phys. 15, 529 (2005).

[16] H. A. Bethe and E. E. Salpeter, Quantum Mechanics of Oneand Two-Electron Atoms (Springer Verlag, Berlin, 1957).

[17] M. Klaiber, K. Z. Hatsagortsyan, and C. H. Keitel, Phys. Rev. A 73, 053411 (2006).

[18] H. R. Reiss, J. Opt. Soc. Am. B 7, 574 (1990).

[19] F. H. M. Faisal and T. Radozycki, Phys. Rev. A 47, 4464 (1993).

[20] L. S. Cederbaum and M. Basler, Phys. Rev. Lett. 103, 133001 (2009).

[21] T. Zuo, A. D. Bandrauk, and P. B. Corkum, Chem. Phys. Lett. 259, 313 (1996). 